\title{
Reconstruction of recycling flux from synthetic camera images, evaluated for the Wendelstein $7-\mathrm{X}$ startup limiter
}

\author{
H Frerichs ${ }^{1}$, F Effenberg ${ }^{1}$, Y Feng ${ }^{2}$, O Schmitz ${ }^{1}$, L Stephey ${ }^{3}$, D \\ Reiter $^{4}, \mathbf{P}$ Börner ${ }^{4}$ and the W7-X team \\ ${ }^{1}$ Department of Engineering Physics, University of Wisconsin - Madison, Madison, \\ WI 53706, USA \\ ${ }^{2}$ Max-Planck-Institut für Plasmaphysik, D-17491 Greifswald, Germany \\ ${ }^{3}$ University of Wisconsin - Madison, HSX Plasma Laboratory, Madison, WI 53706, \\ USA \\ ${ }^{4}$ Forschungszentrum Jülich GmbH, Institut für Energie- und Klimaforschung - \\ Plasmaphysik, D-52425 Jülich, Germany \\ E-mail: hfrerichs@wisc.edu
}

\begin{abstract}
The interpretation of spectroscopic measurements in the edge region of high-temperature plasmas can be guided by modeling with the EMC3-EIRENE code. A versatile synthetic diagnostic module, initially developed for the generation of synthetic camera images, has been extended for the evaluation of the inverse problem in which the observable photon flux is related back to the originating particle flux (recycling). An application of this synthetic diagnostic to the startup phase (inboard) limiter in Wendelstein 7-X (W7-X) is presented, and reconstruction of recycling from synthetic observation of $H_{\alpha}$ emission is evaluated. It is shown that recycling - in terms of re-ionization within the view cone of the camera - is underestimated by a factor of about 3 at the peak location and overestimated in between, and that $H_{\alpha}$ emission related to molecular dissociation can locally yield an additional factor of 2 for the ionization to photon conversion. Fitted conversion factors are extracted from the simulation results which include a dependence on the plasma density.
\end{abstract}

(Some figures in this article are in colour only in the electronic version)

\section{Introduction}

Spectroscopic measurements for the analysis of the edge region are ubiquitous in hightemperature plasmas $[1,2,3]$ with applications in stellarators (e.g. W7-AS [4, 5], W7-X [6, 7], LHD [8, 9], HSX [10, 11]) and tokamaks (e.g. DIII-D [12, 13], NSTX $[14,15]$, TEXTOR-DED $[16,17])$, yet their interpretation in terms of local quantities can be challenging due to line of sight integration effects. The reconstruction of local quantities from these non-local measurements requires additional information that is experimentally very difficult to determine, but which modeling can provide. One 
Reconstruction of recycling flux from synthetic camera images, evaluated for the Wendelstein $7-X$ startup lim

particular application in fusion plasmas is the characterization of particle fluxes and erosion on limiter or divertor targets inferred from observations by a camera with an appropriate filter. At W7-X, such a diagnostic system has been dedicated to the analysis of the startup limiter during the first operation phase (OP1.1) from observation of $H_{\alpha}$ emission $(\lambda=656.2 \mathrm{~nm})[6,7]$. These measurements are based on the "S/XB formalism" $[18,2]$

$$
\Gamma=\mathcal{S}_{\mathcal{X} \mathcal{B}} \cdot \Phi
$$

which relates the measured photon flux $\Phi$ to the particle flux onto the limiter $\Gamma$ through an appropriate choice of conversion factor $\mathcal{S}_{\mathcal{X} \mathcal{B}}$. The first challenge for the evaluation of measurements according to (1) is that the observed photon flux

$$
\Phi(\mathbf{x})=\int_{L_{\mathbf{x}}} d s \cdot \varepsilon(\mathbf{y}(s)), \quad \mathbf{x} \in B=\partial V
$$

related to point $\mathbf{x}$ on the boundary surface is a line-of-sight integral through the plasma volume $V$ rather than a local quantity as $\Gamma(\mathbf{x})$. Here, $L_{\mathbf{x}}$ is the line-of-sight from the camera to point $\mathbf{x}$ and $\varepsilon(\mathbf{y}), \mathbf{y} \in V$ is the local photon emission rate. The ionization per emitted photon coefficient $\mathrm{S}_{\mathrm{XB}}$ facilitates conversion of $\varepsilon(\mathbf{y})$ into a local ionization rate (unlike $\mathcal{S}_{\mathcal{X B}}$ in (1))

$$
\Sigma(\mathbf{y})=\mathrm{S}_{\mathrm{XB}} \cdot \varepsilon(\mathbf{y}),
$$

and the second challenge is that $\Sigma(\mathbf{y})$ still needs to be related to the actual quantity of interest $\Gamma(\mathbf{x})$. This relation exists since recycling neutral particles leave the surface and enter the plasma volume where they are re-ionized. However, some neutral particles may escape the view cone of the camera while others, originating from recycling outside the view cone, may enter. This transport can be summarized by the propagator $T(\mathbf{x}, \mathbf{y})$ which relates the location of re-ionization $\mathbf{y} \in V$ in the plasma volume $V$ to the origin $\mathbf{x} \in B=\partial V$ of the recycled neutral particle on the solid boundary. The resulting ionization rate from recycled particles can then be written as

$$
\Sigma(\mathbf{y})=\int_{B} d \mathbf{x} \cdot \Gamma(\mathbf{x}) \cdot T(\mathbf{x}, \mathbf{y})
$$

without going into detail about the transport model behind $T(\mathbf{x}, \mathbf{y})$ at this point. This dependence on the quantity of interest $\Gamma(\mathbf{x})$ can be exploited in (3) together with (2) to obtain the following expression for the photon flux:

$$
\Phi(\mathbf{x})=\int_{L_{\mathbf{x}}} d s \cdot \frac{1}{\mathrm{~S}_{\mathrm{XB}}(\mathbf{y}(s))} \int_{B} d \mathbf{x}^{\prime} \cdot \Gamma\left(\mathbf{x}^{\prime}\right) \cdot T\left(\mathbf{x}^{\prime}, \mathbf{y}(s)\right) .
$$

Obviously, without detailed information on plasma conditions (or significantly simplifying assumptions), it is not possible to solve (5) for $\Gamma(\mathbf{x})$, yet exactly this is required for the interpretation of measurements.

This paper is focused on evaluating assumptions which are implicit to (and may lead to misinterpretation of) camera data according to (1). Based on self-consistent modeling 
results, we can calculate an appropriate conversion factor $\mathcal{S}_{\mathcal{X B}}$ in (1) for each camera pixel. While such an overall conversion factor may suffice for the direct interpretation of camera data in terms of recycling flux, it does not provide information about the underlying physics. Therefore, we introduce an unfolded version of (5)

$$
\Phi(\mathbf{x})=\frac{1}{\mathrm{~S}_{\mathrm{XB}_{\mathbf{x}}}^{*}} \cdot G_{\mathrm{x}}^{*} \cdot T_{\mathbf{x}}^{*} \cdot \Gamma(\mathbf{x})
$$

where the terms $\mathrm{S}_{\mathrm{XB}_{\mathbf{x}}}^{*}, G_{\mathbf{x}}^{*}$ and $T_{\mathbf{x}}^{*}$ (to be discussed in detail later) represent effective ionization to photon conversion, geometry and transport effects, respectively. The advantage of our approach in (6) is that we can separate and analyze contributions from different physical processes (transport vs. ionizatin/photon emission) within the camera geometry, which are all combined in the overall conversion factor $\mathcal{S}_{\mathcal{X} \mathcal{B}}$ in (1). In particular, this allows us to analyze contributions which go beyond the traditional $\mathrm{S} / \mathrm{XB}$ framework but which need to be included in $\mathcal{S}_{\mathcal{X B}}$.

Wendelstein 7-X (W7-X) has been equipped with a dedicated visible imaging and filterscope system $[7,6]$ for the spatially resolved and integral analysis of particle fluxes (henceforth referred to as recycling) on the inboard limiter during its first operational phase (OP1.1). In order to analyze the process of reconstructing the recycling flux from (5) and to evaluate the uncertainties that can be expected by interpreting experimental data according to (1), we rely on plasma edge modeling. Modeling of the plasma edge provides spatially resolved data for densities and temperatures as well as photon emission rates and recycling on the limiter, and thus facilitates a benchmark of the reconstruction process. We begin with a brief introduction of the computational plasma edge model EMC3-EIRENE and its application to W7-X in section 2. This will provide the foundation for the following analysis. The diagnostic setup for recycling analysis in

W7-X is introduced, and its synthetic implementation is described in section 3. The "forward calculation" (i.e. the calculation of line-of-sight integrals to obtain a synthetic camera image) is a well-defined problem which will provide input for the reconstruction of recycling. The key part of this paper is section 4 in which the "backward calculation" (i.e. the reconstruction of recycling from synthetic camera images) is analyzed in detail. The generic form (6) will be used to separate contributions from transport, surface geometry and atomic/molecular effects for recycling on the inboard limiter in W7-X. While spatial resolution is important for subsequent analysis of erosion on the limiter, information on the global particle balance is obtained from the integrated observation which may require fewer assumptions than the reconstruction of the local recycling flux. We will address this in section 5 .

\section{Modeling of the plasma boundary}

The inherent non-axisymmetric nature of stellarators requires a three-dimensional model for the plasma edge such as EMC3-EIRENE [19, 20]. Initially developed and benchmarked for W7-X's predecessor W7-AS [21, 22, 23], its strength is its 
flexible implementation of configuration space geometry [24, 25]. This flexibility allows application to many present day fusion experiments, and thus facilitates cross machine and even cross configuration type (stellarator vs. tokamak) comparisons of divertor operation [26, 27]. The code is based on a field-aligned coordinate system which is reconstructed from a finite flux tube grid $[22,25]$. While this has to be generated in a pre-processing step, it separates the plasma edge model from the underlying magnetic configuration model.

\subsection{The magnetic configuration}

We apply an idealized coil configuration in order to exploit the $n=5$ toroidal symmetry of W7-X, and we neglect any contributions from error fields due to coil misalignments or equilibrium effects (because the heat loads fit the prediction in the ideal configuration quite well [28, 29] even though small error fields are present [30, 31])). Stellarator symmetry then allows to focus the simulations on a toroidal domain of $\Delta \varphi=36$ deg. During the first operation phase, W7-X is operated with five inboard limiters at the bean shaped symmetry plane [32] (see e.g. figures 1 and 3 in this reference). For this, the rotational transform has been reduced in order to shift the $5 / 5$ magnetic island of the "standard case" magnetic configuration far out and retain good flux surfaces at the position of the limiter. The resulting scrape-off layer (SOL) is a mix of three types of flux bundles due to the magnetic shear and the toroidal and poloidal localization of the limiter. These flux bundles have a connection length of 1, 1.2 and 2.2 toroidal turns (see e.g. figure 2 in reference [28]), i.e. they connect back to the same limiter after one turn, or connect to the next limiter after one or two turns.

\subsection{The edge plasma and neutral gas model}

Steady state edge plasma and neutral gas conditions are modeled with the EMC3EIRENE code. EMC3 implements a fluid model for the edge plasma including trace impurities (for a detailed introduction to the model equations see reference [33] or [34], key features are summarized below). Particle, parallel momentum and electron and ion energy balance equations are solved for the main plasma species. Resulting quantities provided by EMC3 are the plasma density $n_{i}=n_{e}$, the Mach number $M$ for the plasma velocity along field lines, and the electron and ion temperatures $T_{e}$ and $T_{i}$, respectively. The parallel electric field $E_{\|}$is obtained from the electron momentum balance neglecting terms proportional to the electron mass $m_{e}$. A full particle balance equation is solved for each impurity charge state $Z$ including ionization and recombination into adjacent charge states. The corresponding velocity $v_{Z}$ along field lines is calculated from a local force balance between friction with main ions and electrical, impurity pressure and thermal forces. Equal ion temperature $T_{Z}=T_{i}$ is assumed for all impurities. Impurity (carbon) production is due to sputtering related to the incident ion flux, and - in the present simulations - is approximated by a user defined sputter coefficient $C=2 \%$. Production and transport of hydrocarbon is neglected at this point. 
Reconstruction of recycling flux from synthetic camera images, evaluated for the Wendelstein 7 -X startup lin

Classical transport (Braginskii) is assumed along magnetic field lines, while "anomalous" cross-field transport is taken into account by free model parameters $D_{\perp}$ for particle transport and $\chi_{e \perp}$ and $\chi_{i \perp}$ for electron and ion energy transport, respectively (cross-field viscosity is implicitly set to $\eta_{\perp}=m_{i} n_{i} D_{\perp}$ in the code). Cross-field drifts such as $\mathbf{E} \times \mathbf{B}$ are presently neglected in EMC3, as well as kinetic corrections to the fluid model (so called "flux limits"). Sound speed and sheath heat transmission coefficients are applied at boundary elements, and source terms in the balance equations are due to interaction with neutral gas (excitation, ionization, charge exchange).

Solid surfaces provide a sink for the plasma: electrons and ions recombine on the surface and are re-emitted (i.e. recycled) as neutral particles. Presently, absorption is neglected and $100 \%$ of the incident flux is recycled. EMC3 is coupled to the Monte Carlo transport solver EIRENE [35, 36] for interaction with neutral gas. Both atomic and molecular hydrogen is included in EIRENE, and EIRENE relies on the TRIM database [37] for the surface reflection model. Atomic and molecular processes that are taken into account in the present simulations are

$$
\begin{array}{lll}
H+e^{-} & \rightarrow H^{+}+2 e^{-} & \text {(el. impact ionization) } \\
H^{+}+H & \rightarrow H+H^{+} & \text {(charge exchange) } \\
e^{-}+H_{2} \rightarrow e^{-}+H+H & \text { (dissociation) } \\
e^{-}+H_{2} \rightarrow 2 e^{-}+H_{2}^{+} & \text {(non-dissociative ionization) } \\
e^{-}+H_{2} \rightarrow 2 e^{-}+H+H^{+} & \text {(dissociative ionization) } \\
e^{-}+H_{2}^{+} \rightarrow 2 e^{-}+2 H^{+} & \text {(dissociative ionization) } \\
e^{-}+H_{2}^{+} \rightarrow e^{-}+H+H^{+} & \text {(dissociative excitation) } \\
e^{-}+H_{2}^{+} \rightarrow H+H & \text { (dissociative recombination) }
\end{array}
$$

based on the AMJUEL database [38, 39]. Furthermore, EIRENE can provide the resulting $H_{\alpha}$ emission rates, from which synthetic camera images can be generated. A self-consistent solution for the edge plasma - neutral gas mix is obtained by iterating between the two codes.

Most of the following synthetic images are based on a reference simulation in which the density at the last closed flux surface has been set to $n_{\text {sepx }}=2 \cdot 10^{18} \mathrm{~m}^{-3}$ as a control parameter and the heating power has been set to $P_{\text {in }}=2 \mathrm{MW}$. These parameters have been chosen to provide an edge plasma which is in the ballpark of experimental conditions [40]. Cross-field transport has been set to $D_{\perp}=1 \mathrm{~m}^{2} \mathrm{~s}^{-1}$ and $\chi_{e \perp}=\chi_{i \perp}=3 D_{\perp}$ which provides good agreement with heat load observations [28, 29]. A sensitivity study for $n_{\text {sepx }}$ is included in a later section. The calculated $H_{\alpha}$ emission in this reference simulation is shown in 1 . It can be seen that the peak emission is located in front of the limiter, because this is where the plasma recycles to neutral gas, and that the emission on the outboard side is several orders of magnitude smaller.

\subsection{The computational domain}

The computational mesh for plasma edge simulations has been divided into two zones: a high toroidal resolution zone near the limiter (10 mesh slices within $\Delta \varphi=2 \mathrm{deg}$ ), 


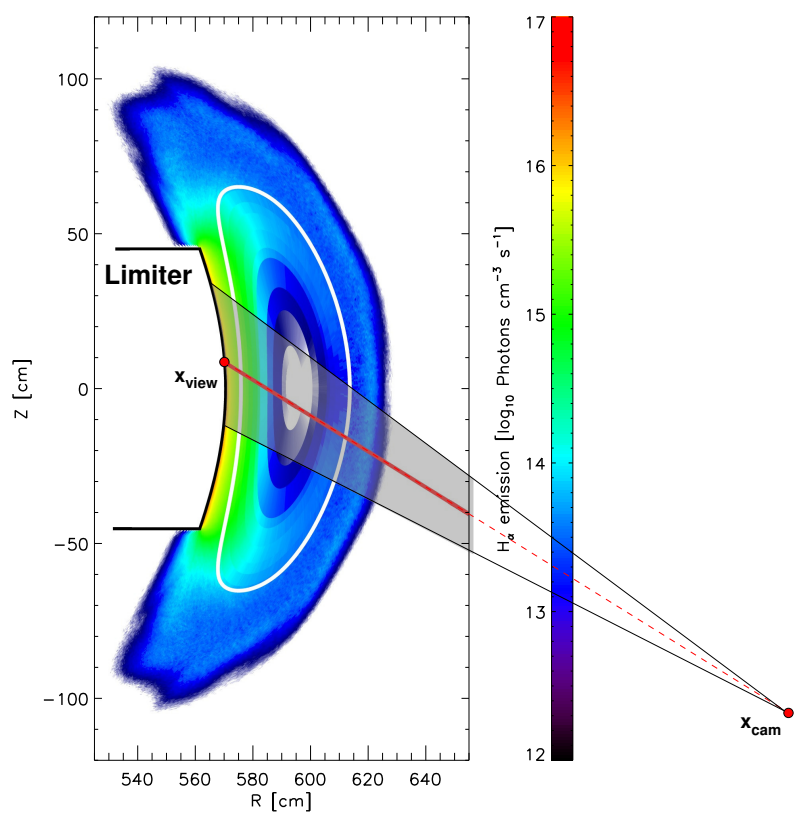

Figure 1. $H_{\alpha}$ emission at the bean shaped symmetry plane with an overlay of the view cone of a camera at the AEA30 port. The core interface in the EMC3 simulations is indicated in white.

and a lower resolution zone away from the limiter (30 mesh slices in the complementary domain). This high toroidal resolution zone is necessary in order to capture the toroidal variation in the synthetic images. Radial and poloidal resolution within each mesh slices is set to $140 \times 673$. The computation domain for EMC3 (edge plasma) is limited to the edge (which traditionally includes the outermost part of the confined region) as indicated by the white outline in figure 1 . However, it can be necessary to include the interaction between the neutral gas and the core plasma. This can be captured in the simulations by an extended computational domain for EIRENE (neutral gas) in which the plasma density and temperature can be set explicitly. In the present setup, the computational grid is extended by 10 additional layers inside the core interface for EMC3. In the following we focus on the generation and interpretation of synthetic camera images based on this data, and we refer to reference [28] for a detailed analysis of plasma conditions and limiter heat loads.

\section{Synthetic camera images}

A versatile synthetic diagnostic module has been recently developed which can capture the realistic three dimensional setup of various plasma edge diagnostics [41]. The synthetic camera setup is defined by 1) its location $\mathbf{x}_{\text {cam }}, 2$ ) either the base view point $\mathbf{x}_{\text {view }}$ or the view direction $\mathbf{v}_{\text {view }}=\mathbf{x}_{\text {view }}-\mathbf{x}_{\text {cam }}$, and 3) the angle of view $\alpha_{\text {view }}$. The angle of view may be given explicitly or implicitly by the size of the observation window in the plane perpendicular to the base line of sight. The field of view is discretized by a 

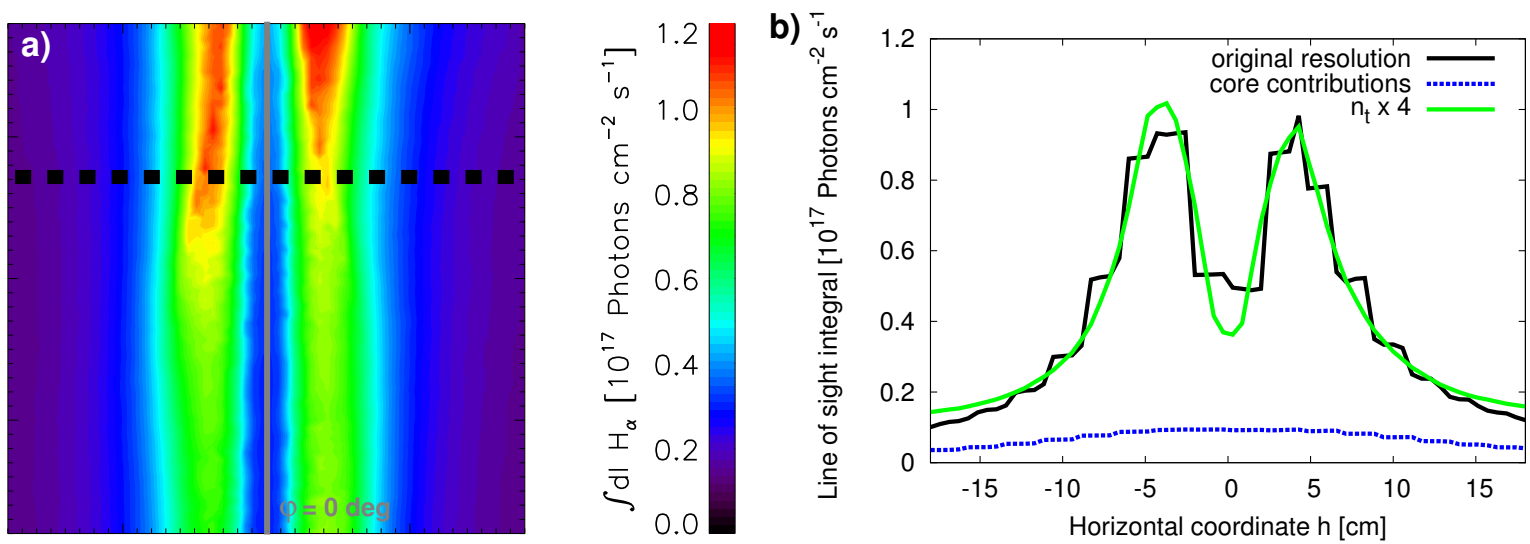

Figure 2. (a) Synthetic image from an $H_{\alpha}$ filtered camera at port AEA30, generated from a grid with high toroidal resolution around the limiter $\left(n_{t} \times 4\right)$, (b) profiles along the horizontal coordinate as indicated by the dashed line on the left.

number of pixels, and each pixel $(i, j)$ defines an individual line of sight $L_{i j}$. A synthetic camera image for a selected line emission is generated by calculating the line of sight integral

$$
\Phi_{i j}=\int_{L_{i j}} d s \varepsilon(s)
$$

for each pixel, where $\varepsilon(s)$ is the local photon emission rate along the line of sight. Numerical integration of (7) requires a discretization of each line of sight. An earlier implementation [42] was based on sampling of $\varepsilon$ at a number of points along $L_{i j}$, and this discretization had to be generated manually with particular care on the resolution. The new implementation of (7), on the other hand, is facilitated by the discretized representation of data from EMC3-EIRENE, i.e. the photon emission rates are provided as cell averaged values (i.e. $\varepsilon(s)=\varepsilon_{k}$ in cell $k$ ). Thus, within the given data representation, (7) is equivalent to

$$
\Phi_{i j}=\sum_{k=1}^{n_{i j}} \Delta s_{k} \cdot \varepsilon_{k}
$$

where $n_{i j}$ is the number of cells through which this particular line of sight passes, and $\Delta s_{k}$ is the length of the segment in the corresponding cell. This discretization is generated by tracing a virtual neutral particle in the EMC3-EIRENE mesh, which automatically provides the optimal resolution so that (8) gives the exact value of the line of sight integral (for the given set of discretized plasma edge data).

\subsection{Implementation of a synthetic camera at port AEA30}

A visible camera for a direct view on the limiter is located at the AEA30 port behind a large diameter (184 $\mathrm{mm}$ clear aperture) uncoated sapphire window at a distance of $3.2 \mathrm{~m}$ from the limiter. The field of view is indicated in figure 1 and corresponds to a 


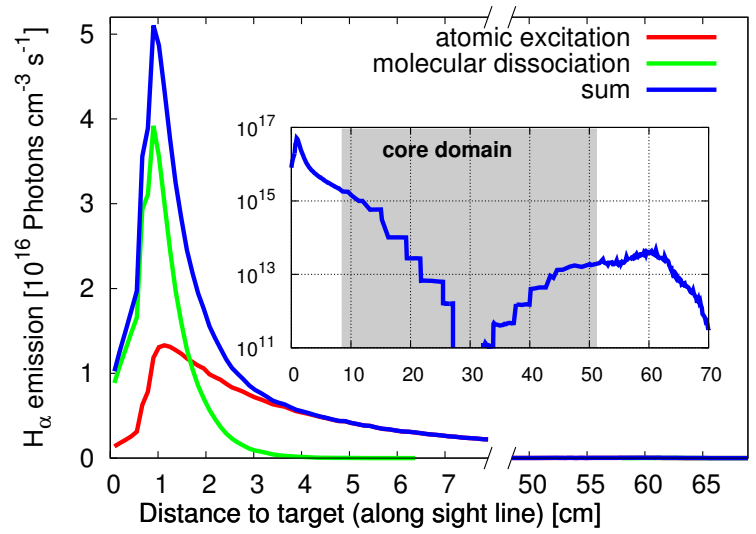

Figure 3. Contributions to the $H_{\alpha}$ emission from excitation (red) and molecular dissociation (green) along the line of sight for a selected pixel. The inset shows the full profile including the core domain on a logarithmic scale.

window of $36 \times 36 \mathrm{~cm}$ in the observation plane at the base point $\mathbf{x}_{\mathrm{view}}$ perpendicular to the base view line (red). The resolution of $1024 \times 1024$ pixel allows a spatial resolution of the limiter below $1 \mathrm{~mm}$. The camera is equipped with an $H_{\alpha}$ filter which was later found be substantially red-shifted centered at $659.4 \mathrm{~nm}$ with a FWHM of $4 \mathrm{~nm}$ (i.e. the filter favors transmission of the C-II line at $658.2 \mathrm{~nm}$ by almost one order of magnitude which may contaminate the measurement). For more details about the experimental setup we refer to reference [7].

A synthetic camera image is shown in figure 2 (a), where the horizontal coordinate is located in the toroidal direction (i.e. perpendicular to figure 1), and the vertical coordinate in the image is located within the plane of figure 1 perpendicular to the base line of sight. The center point in the synthetic camera image corresponds to the base point $\mathbf{x}_{\text {view }}$ in the bean shaped symmetry plane. Such a smooth camera image can be generated after the toroidal resolution is increased by a factor of 4 . However, as indicated in figure 2 (b), the dominant features are already captured within the original grid resolution. Increasing the radial and poloidal resolution by a factor of 2 has virtually no impact on the resulting camera image.

\subsection{Distribution along line of sight}

Profiles along a selected line of sight are shown in figure 3 . The local $H_{\alpha}$ emission can be split in contributions from excitation of atomic hydrogen (red) and contributions related to the dissociation of molecular hydrogen (green). It can be seen that contributions from molecular dissociations dominate within the first $15 \mathrm{~mm}$ in front of the limiter, which accounts for $43 \%$ of the integral value. Beyond $30 \mathrm{~mm}$, these contributions become negligible, but the contributions from atomic excitation remain finite even at the coreinterface of the edge plasma. Beyond that, the core domain contributes $15 \%$ to the integral value. These values depend on the edge plasma in front of the limiter, and on 
the plasma conditions that have been set for the core plasma. In particular, a smooth continuation of the photon emission profile into the core requires to define a smooth density and temperature profile in the core. In the present case, this continuation has been guided by measurements from the Thomson scattering system, but it would need to be adapted for density and heating power scans. Furthermore, results for the neutral gas in the core domain (where cells are usually merged to one macro cell for each radial layer) have to be resolved in poloidal and toroidal direction for the generation of synthetic camera images. However, since this step does not affect the edge plasma calculation in EMC3, one EIRENE iteration on the fine grid may be done as a postprocessing step on a converged solution. On the other hand, the leading contributions from the core region may be estimated by fitting an exponential to the tail in the edge region. Such a fit to the total $H_{\alpha}$ emission on $\delta l=1 \mathrm{~cm}$ before the core interface provides a contribution of $12 \%$.

\subsection{Characterization of synthetic image}

The dominant feature in the synthetic camera image (figure $2(\mathrm{a})$ ) is the dual vertical stripe which one stripe located left and right of the symmetry plane at $\varphi=0 \mathrm{deg}$, respectively. Field lines intersect the limiter to the left and right of the symmetry plane, while field lines are tangential to the limiter surface at the symmetry plane itself. Thus, two vertical stripes can be expected, and they are confirmed in experimental observations (see figure 2 in [6]). A secondary feature is the vertical intensity variation in the synthetic image which can be explained by the geometry of the view cone: the line of sight from the camera intersects the limiter at a more shallow angle towards the top, and this results in longer segment through the neutral gas cloud. For a poloidally homogeneous gas cloud, the integral value can be expected to scale with $1 / \cos \gamma$ (where $\gamma$ is the incident angle toward the surface normal, see figure 4), which for the given setup accounts for an increase of $40 \%$ between bottom and top as seen in the synthetic image. Experimental observations, however, show the opposite trend: the intensity is higher at the bottom of the picture.

While the primary characteristic is recovered in the synthetic image, we need to include include a short discussion of possible reasons for the top/bottom mismatch between synthetic and experimental images which could be related to 1) perturbations of the camera image, (e.g. by impurity radiation and/or reflections on the limiter from light sources at other locations) and/or 2) deviations in recycling and the resulting $H_{\alpha}$ emission itself (e.g. due to mismatching plasma conditions related to transport assumptions). Analysis of the heat load patterns has shown reasonable agreement with predictions by EMC3-EIRENE [28, 29], which suggest that particle loads onto the limiter should exhibit a similar pattern. Of considerable concern are perturbations from impurities which can contaminate the experimental observations because of the red-shifted transmission curve of the filter. Simulations with trace impurities (carbon) indicate that contributions from the C-II line at $658.2 \mathrm{~nm}$ are not dominant, but this 


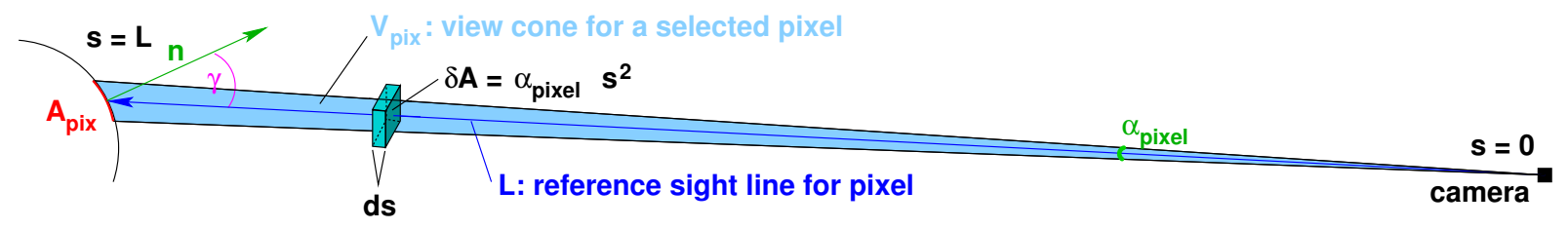

Figure 4. Sketch of the view cone for a given pixel. The finite angle of view $\alpha_{\text {pixel }}$ results in an observation area $A_{\text {pix }}$ on the boundary surface.

could be related to simplifications in the impurity model and may require to resolve different impurity production processes (physical vs. chemical sputtering with formation and dissociation of hydrocarbons) and to include a full (kinetic) transport model for the low charge state ions, as well as to cover all possible line emission processes within the transmission curve. A major concern is also that $H_{\alpha}$ emission occurs at the very edge of the transmission curve of the filter: a small shift of the transmission curve depending on the optical path related to a pixel may result in a large variation. In particular, a similar filter was later found to exhibit a small shift in the transmission curve depending on the incident angle on the filter, which results in an increase of $H_{\alpha}$ transmission by a factor of 2.7 at a tilt angle of $2.3 \mathrm{deg}$ compared to normal incident. Finally, we find that synthetic images for a camera setup at an AEF10-like port show a better qualitative agreement with experimental observations, and the partial overlap between the two systems may be used to cross-calibrate experimental data. Because of the issue with the filter, we defer benchmarking of experimental data and modeling results to the next operation phase in which new filters will be applied. In the following we will look at the relation between recycling as the quantity of interest and the observable photon emission based on self-consistent data (from modeling), and by that address requirements for the reconstruction of recycling.

\section{Spatially resolved reconstruction of recycling}

Recycling on limiter and divertor targets is often inferred from spectroscopic observations based on (1) by exploiting the relation between ionization and photon emission. However, this implies that the photons emitted by recycled neutral particles in the view cone $V_{\text {pix }}$ of a pixel (see sketch in figure 4) are related to the recycling flux $\Gamma_{\text {rec }}$ on the boundary surface, and that a conversion factor for the integral photon flux can be based on local S/XB values. The former assumption is hardly fulfilled, it is likely that particles recycled on the observed area $A_{\text {pix }}$ on the boundary surface are re-ionized outside the view cone $V_{\text {pix }}$, and that other recycled particles enter the view cone and are re-ionized within. The true relation (5) between the observable photon flux and the recycling flux is far more complicated than (1), and evaluation of experimental data within the latter relies on simplifying assumptions which may lead to misinterpretation of these measurements. In the following we will separate the contributions from different processes, and analyze their impact on reconstruction of recycling on the W7-X startup 

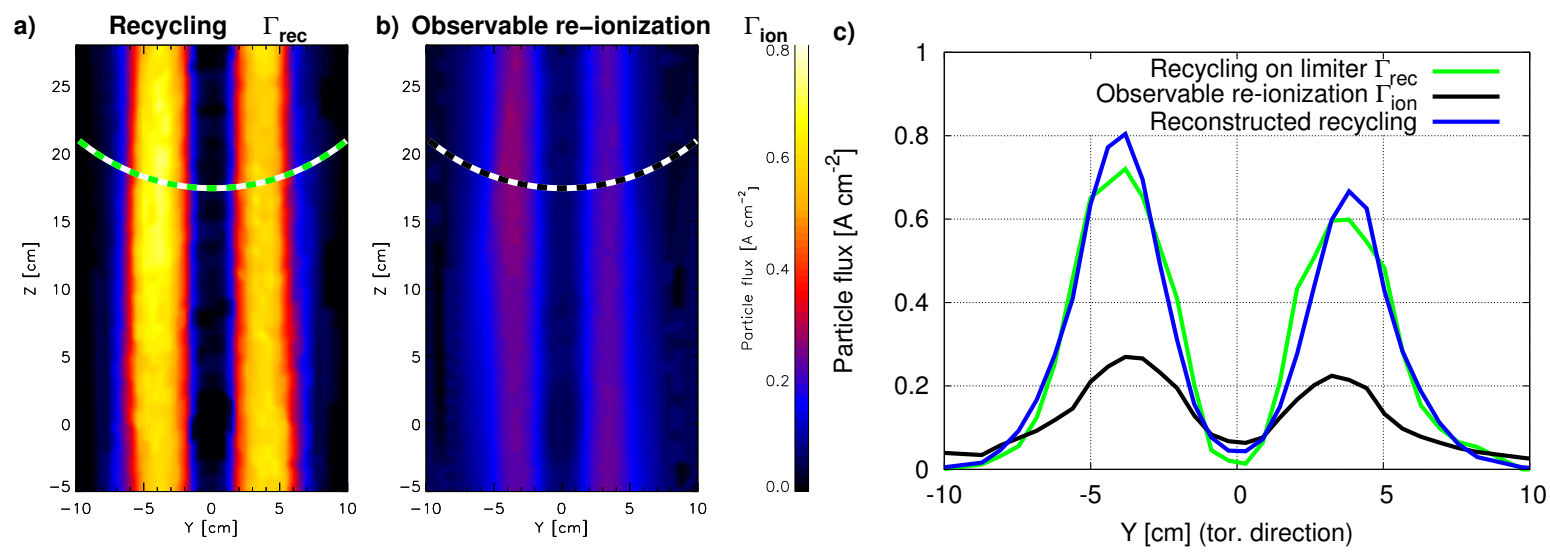

Figure 5. (a) Recycling $\Gamma_{\text {rec }}$ on the limiter, (b) observable re-ionization $\Gamma_{\text {ion }}$ in the view cone of the camera at AEA30 mapped to the limiter, (c) selected profiles across the limiter as indicated by the dashed lines on the left.

limiter.

\subsection{Recycling to re-ionization conversion}

Let us begin by relating the recycling flux $\Gamma_{\text {rec }}$ to the observable re-ionization $Q_{\text {src }}$ within the view cone. For a given pixel as sketched in figure 4, let

$$
A_{\text {pix }}=\frac{\alpha_{\text {pixel }} L^{2}}{\cos \gamma}
$$

be the observed area on the limiter and $\Gamma_{\text {rec }} \cdot A_{\text {pix }}$ the total recycling flux on the observed area. The observable recycling is defined by the re-ionization within the view cone mapped to the limiter surface:

$$
\Gamma_{\text {ion }}=\frac{1}{A_{\text {pix }}} \int_{V_{\text {pix }}} d V \Sigma
$$

where $\Sigma$ is the local ionization rate. A comparison between the actual and observable recycling is shown in figure 5. It can be seen that the observable re-ionization underestimates recycling by a factor of 3-4 at the peak locations. On the other hand, recycling is overestimated between the peaks. Since both quantities are available from modeling, we can define a self-consistent conversion factor

$$
C_{\Gamma \rightarrow S}=\Gamma_{\text {rec }} / \Gamma_{\text {ion }}
$$

as the local ratio of these quantities. The horizontal dependence of $C_{\Gamma \rightarrow S}$ is shown in figure 6 (a) where each data point represents one pixel of the synthetic camera image. This demonstrates that there is only a weak vertical dependence of $C_{\Gamma \rightarrow S}$. As suggested by figure 5, we find $C_{\Gamma \rightarrow S}>1$ at the recycling peaks and $C_{\Gamma \rightarrow S}<1$ in between. We also find that the overestimation of peak recycling is somewhat smaller at much higher separatrix density (beyond conditions in the experiment). 

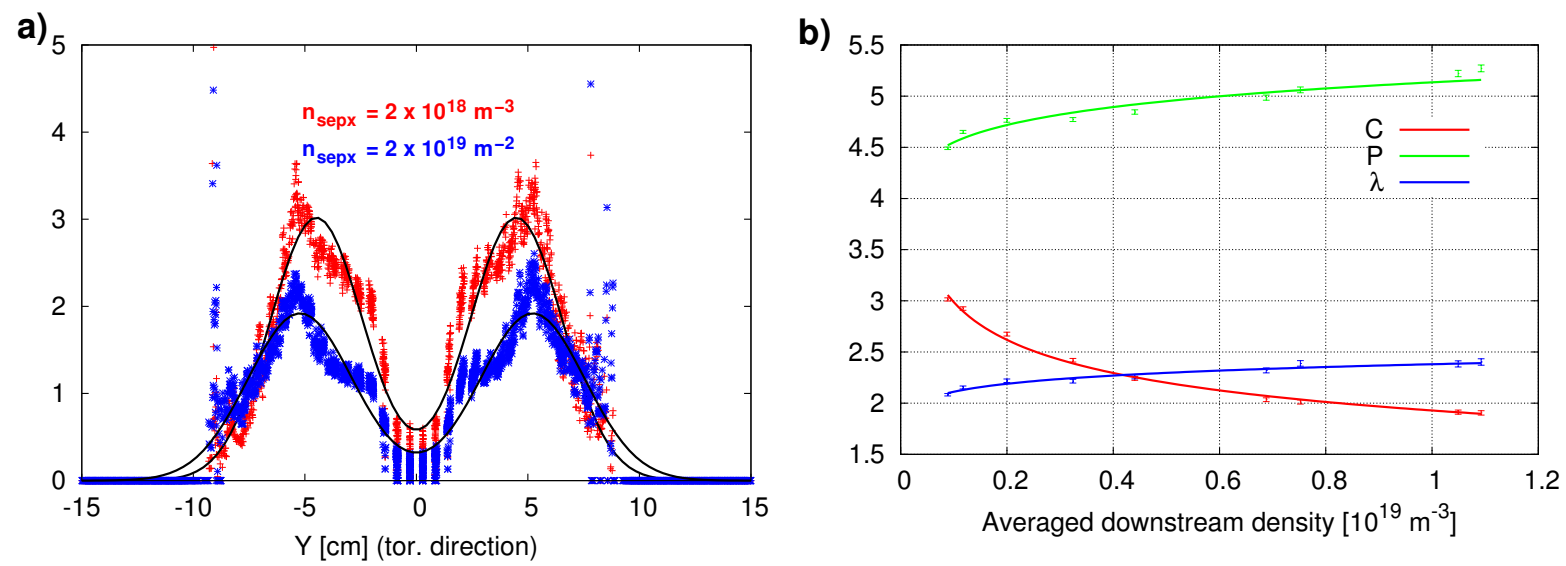

Figure 6. (a) Horizontal dependence of conversion factor $C_{\Gamma \rightarrow S}$ for the reference data set (red) and for a case with increased separatrix density (blue). Each data point represents a pixel in the synthetic camera image; black lines are fits according to (12). (b) Dependence of the fit coefficients on the averaged downstream density.

Evaluation of experimental observations requires application of this conversion factor. This either requires dedicated modeling for each measurement, or an adequate approximation for $C_{\Gamma \rightarrow S}$. In order to facilitate processing of camera images for the reconstruction of recycling, we introduce a fit function that covers the essential spatial dependence of $C_{\Gamma \rightarrow S}$ :

$$
C_{\Gamma \rightarrow S}^{(\text {fit })}(y)=C\left(e^{\frac{-(y-P)^{2}}{2 \lambda^{2}}}+e^{\frac{-(y+P)^{2}}{2 \lambda^{2}}}\right)
$$

with fit parameters $C, P$ and $\lambda$. This double Gaussian fit may not match every detail of $C_{\Gamma \rightarrow S}$ as seen in figure 6 (a), but it is still a reasonable shape and much better than a constant value (of 1 or some manually applied fudge factor). The resulting fit coefficients may be used to characterize $C_{\Gamma \rightarrow S}$ from a given simulation, however, they are not universal parameters. The dependence on plasma conditions is shown in figure 6 (b) for which the limiter averaged downstream density $n_{d}$ has been chosen as control parameter. It can be seen that each coefficient fits a simple power law (solid lines) $A \cdot\left(n_{d}\left[10^{19} \mathrm{~m}^{-3}\right]\right)^{\alpha}$ with weak dependence on $n_{d}$. The parameters $A$ and $\alpha$ for each coefficient are given in the table below:

\begin{tabular}{l|l|l}
$*$ & $A_{*}$ & $\alpha_{*}$ \\
\hline$C$ & $1.929 \pm 0.018$ & $-0.1900 \pm 0.0057$ \\
$P$ & $5.135 \pm 0.044$ & $0.0526 \pm 0.0052$ \\
$\lambda$ & $2.381 \pm 0.019$ & $0.0520 \pm 0.0047$
\end{tabular}

Since the dependence on $n_{d}$ is weak, one may evaluate the coefficients for $C_{\Gamma \rightarrow S}$ based on Langmuir probe measurements for evaluation of experimental camera images. All simulations have been performed for the same heating power $P_{\text {in }}=2 \mathrm{MW}$, however, a separate dependence on the downstream temperature may be included by extending the simulation database. 


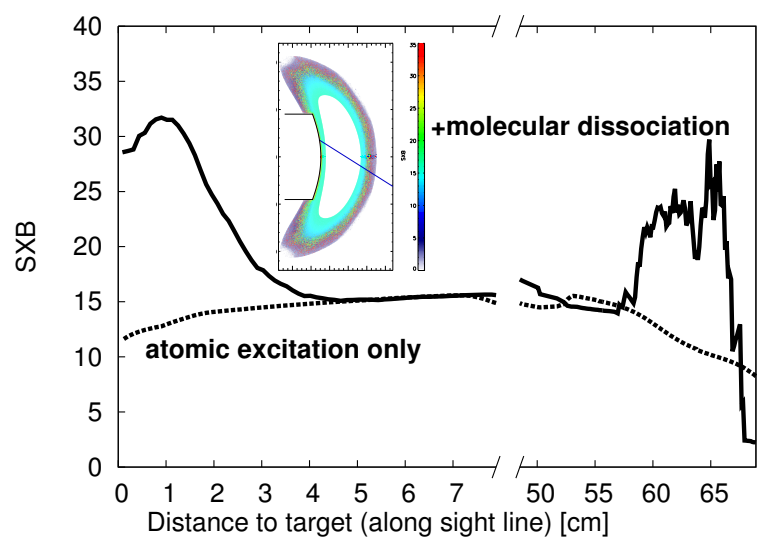

Figure 7. Self-consistent $\mathrm{S} / \mathrm{XB}$ values along a particular line-of-sight based on simulation results for $\Sigma$ and $\varepsilon$ from EIRENE (solid line). For comparison, S/XB values from atomic excitation / ionization only are presented as dashed line. This particular line of sight refers to the green $\mathrm{x}$ in figure 8 (a).

\subsection{Ionization to photon conversion}

The second contribution to $\mathcal{S}_{\mathcal{X} \mathcal{B}}$ in (1) is the actual conversion between ionizations and photon emission. While ionizations per photon coefficients $\mathrm{S}_{\mathrm{XB}}$ are available from databases such as the ADAS project [43, 44], one needs to keep in mind that these may not include all of the relevant processes. E.g. S/XB data for $H_{\alpha}$ often includes only ionization of atomic hydrogen without contributions from molecular dissociation. Both processes are included in the EMC3-EIRENE simulations, and since both the resulting ionisation rate $\Sigma$ and the photon emission rate $\varepsilon$ are modeling results, we can calculate a spatially resolved and self-consistent value $\mathrm{S}_{\mathrm{XB}}=\Sigma / \varepsilon$. This is shown in figure 7 (solid line), which demonstrates that molecular processes can contribute up to a factor of 2. Furthermore, while the traditional (ionization only) $\mathrm{S}_{\mathrm{XB}}$ value for $H_{\alpha}$ is fairly constant (dashed line), it can be seen that the self-consistent value can change along the line of sight of the camera by a factor of 2 . Note, however, that for applications in cold divertors (a few eV), even the traditional $\mathrm{S}_{\mathrm{XB}}$ (e.g. from ADAS) can exhibit larger variations along a line-of-sight. This complicates the analysis in (1) in terms of a global conversion factor $\mathcal{S}_{\mathcal{X} \mathcal{B}}$ : we need the relation between the line of sight integrated quantities rather than local quantities. Therefore, let us define an integrated S/XB value (i.e. conversion factor $C_{\mathrm{S} / \mathrm{XB}}$ ) for each camera pixel by

$$
C_{\mathrm{S} / \mathrm{XB}}=\int_{V_{\text {pix }}} d V \Sigma / \underbrace{\int_{V_{\text {pix }}} d V \varepsilon}_{\equiv Q_{\text {phot,pix }}} .
$$

The horizontal dependence of $C_{\mathrm{S} / \mathrm{XB}}$ is shown in figure 8 (a). It can be seen that $C_{\mathrm{S} / \mathrm{XB}} \approx 23$ at the location of the recycling peaks, which is an average of the peak $\mathrm{S}_{\mathrm{XB}}$ value of about 32 in front of the limiter resulting from molecular dissociation 
Reconstruction of recycling flux from synthetic camera images, evaluated for the Wendelstein 7 -X startup lin
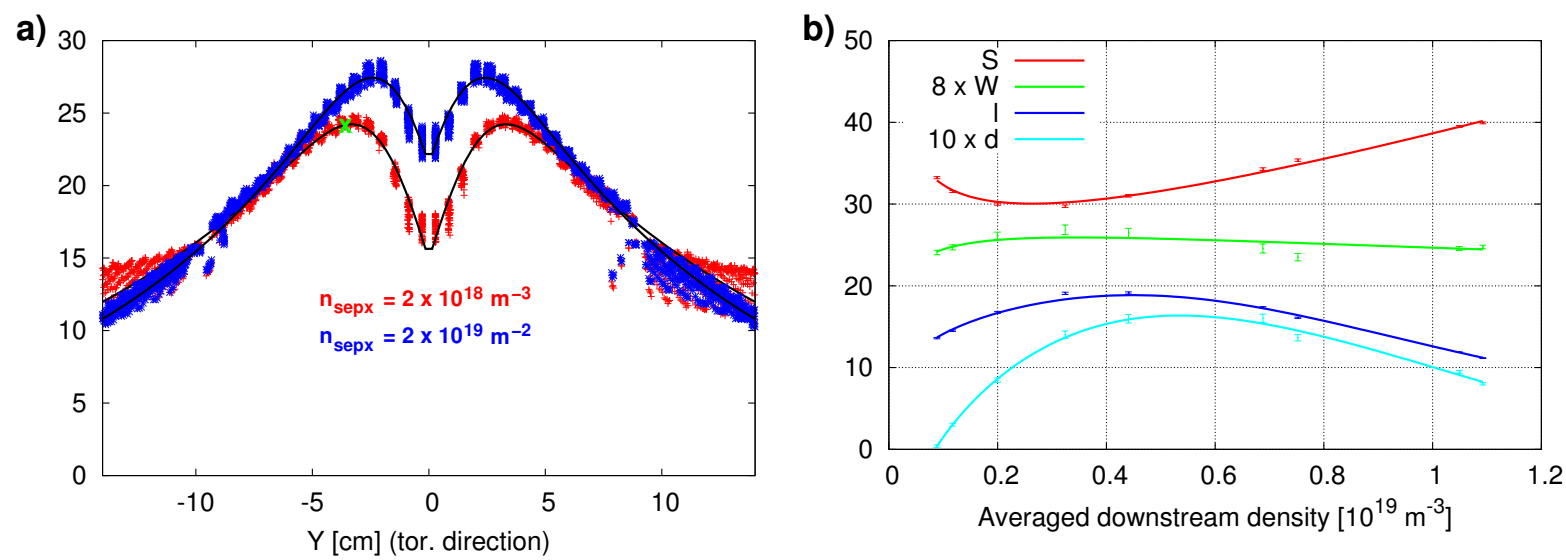

Figure 8. (a) Horizontal dependence of conversion factor $C_{\mathrm{S} / \mathrm{XB}}$ for the reference data set (red) and for a case with increased separatrix density (blue). Each data point represents a pixel in the synthetic camera image; black lines are fits according to (14).

(b) Dependence of the fit coefficients on the averaged downstream density.

and $\mathrm{S}_{\mathrm{XB}} \approx 15$ further inside the plasma (figure 7). Contributions from molecular dissociation are larger at higher density and lower temperature (blue data points in figure 8 (a)), resulting in a larger peak $C_{\mathrm{S} / \mathrm{XB}}$ value. Again, there is almost no vertical dependence of $C_{\mathrm{S} / \mathrm{XB}}$, and we can characterize the data by a fit expression along the horizontal coordinate:

$$
C_{\mathrm{S} / \mathrm{XB}}^{(\mathrm{fit})}(y)=\frac{S}{2} \exp \left[\left(\frac{W}{2 l}\right)^{2}-\frac{|y|+d}{l}\right] \cdot \operatorname{erfc}\left[\frac{W}{2 l}-\frac{|y|+d}{W}\right]
$$

where $S, W, d$ and $l$ are fit coefficients. It can be seen by the black lines in figure 8 (a) that this parametrization fits the results very well. The dependence of the fit coefficients on plasma conditions is somewhat more complicated than the that of the coefficients for $C_{\Gamma \rightarrow S}$ as shown in figure 8 (b). Nevertheless, a functional dependency on $X=n_{d}\left[10^{19} \mathrm{~m}^{-3}\right]$ can still be found:

$$
\begin{array}{rlrl}
S(X) & = & & C_{S} \cdot\left(X^{\alpha_{S}}+X^{\beta_{S}}\right) \\
W(X) & = & C_{W} /\left(X^{\alpha_{W}}+X^{\beta_{W}}\right) \\
l(X) & = & C_{l} /\left(X^{\alpha_{l}}+X^{\beta_{l}}\right) \\
d(X) & =B_{d}+C_{d} /\left(X^{\alpha_{d}}+X^{\beta_{d}}\right)
\end{array}
$$

\begin{tabular}{l|l|l|l|l}
$*$ & $B_{*}$ & $C_{*}$ & $\alpha_{*}$ & $\beta_{*}$ \\
\hline$S$ & & $19.324 \pm 0.090$ & $-0.199 \pm 0.005$ & $1.037 \pm 0.044$ \\
$W$ & & $6.166 \pm 0.072$ & $-0.208 \pm 0.033$ & $0.397 \pm 0.120$ \\
$l$ & & $25.205 \pm 0.147$ & $-0.251 \pm 0.006$ & $2.717 \pm 0.097$ \\
$d$ & $-2.020 \pm 0.268$ & $6.054 \pm 0.519$ & $-0.443 \pm 0.017$ & $1.741 \pm 0.210$
\end{tabular}




\subsection{Reconstruction of recycling unfolded}

So far, we have related recycling to re-ionization in the view cone, and the ionization to the photon emission in the view cone. Before we can combine the results from sections 4.1 and 4.2 for an unfolded version of (5), we need to relate the integrated volumetric photon emission $Q_{\text {phot,pix }}$ to the observable photon flux $\Phi$ at the location of the camera which takes into account the Etendue of the optical system (see appendix): $Q_{\text {phot,pix }}=\alpha_{\text {pixel }} L^{2} G \Phi$ where $G \approx 1$. Now we can combine (11) and (10) for the observable re-ionization with (13) for the photon emission and (9) for the observed area:

$$
\Gamma_{\text {rec }}=\underbrace{C_{\Gamma \rightarrow S} \cdot C_{\mathrm{S} / \mathrm{XB}} \cdot G \cdot \cos (\gamma)}_{=\mathcal{S}_{\mathcal{X B}}} \cdot \Phi
$$

Thus, we have related recycling $\Gamma_{\text {rec }}$ to observable photon flux $\Phi$ via re-ionization and photon emission within the view cone as indicated in (6). Note that from figures 6 and 8 we find minimal vertical dependence of $C_{\Gamma \rightarrow S}$ and $C_{\mathrm{S} / \mathrm{XB}}$, i.e. the dominant effect in the top to bottom variation in the synthetic image is given by the incident angle of the camera view.

Dedicated modeling of a particular scenario with EMC3-EIRENE requires considerable computational resources and is not viable for post-pulse processing. However, a database covering several density and temperature combinations can be set up before a campaign from which fitted conversion factors $C_{\mathrm{S} / \mathrm{XB}}{ }_{\text {(fit) }}$ and $C_{\Gamma \rightarrow S}{ }_{\Gamma \rightarrow}^{(\mathrm{fit})}$ can be generated. Replacing $C_{\mathrm{S} / \mathrm{XB}}$ and $C_{\Gamma \rightarrow S}$ in (16) by their fitted counterparts from (14) and (12), respectively, facilitates a smooth reconstruction of the recycling flux from spectroscopic observations which can be done on a much shorter timescale, possibly in post-pulse processing. This reconstructed recycling flux is shown in blue in figure 5 (c) along with the original recycling.

\section{Evaluation of global particle balance}

In the previous section we have shown that a spatially resolved reconstruction of recycling based on spectroscopic observations alone is challenging. Even though conversion factors for the local recycling flux have been obtained from self-consistent modeling, they depend on plasma conditions and would likely need to be updated with advancements in the edge plasma model. Reconstruction of the global particle balance does not require knowledge of the exact spatial distribution of recycling, re-ionization and photon emission. Thus, reconstruction of the global particle balance may be more feasible as long as the relevant processes happen within the view cone of the camera. In analogy to (11) we can write the integral version of the recycling to re-ionization conversion as

$$
\overline{C_{\Gamma \rightarrow S}}=\int_{A_{\text {cam }}} d A \Gamma_{\text {rec }} / \int_{V_{\text {cam }}} d V \Sigma
$$


Reconstruction of recycling flux from synthetic camera images, evaluated for the Wendelstein $7-X$ startup lim
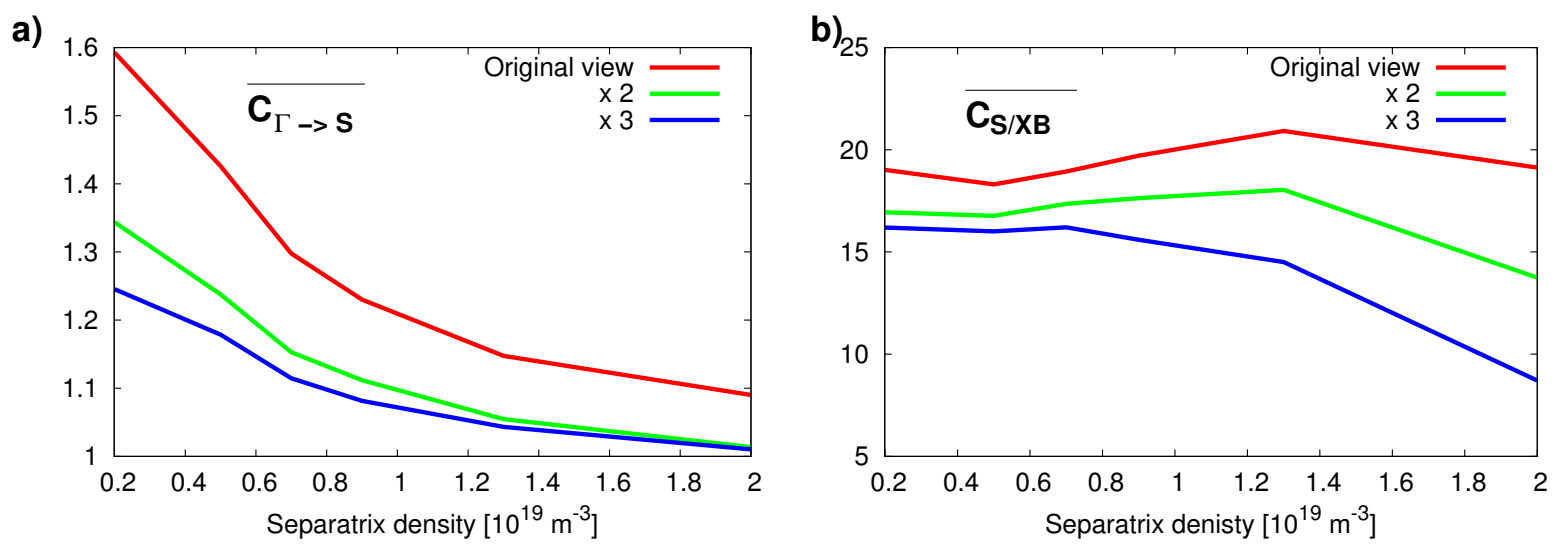

Figure 9. Camera view integrated conversion factors $\overline{C_{\Gamma \rightarrow S}}$ from (17) (a) and $\overline{C_{\mathrm{S} / \mathrm{XB}}}$ from (18) (b). A larger view cone by a factor of two (green) and three (blue) is explored.

where the integrals have to be calculated over the total view cone of the camera $V_{\text {cam }}$ and the corresponding observed area on the limiter $A_{\text {cam }}$. Both integrals are evaluated as the sum over all pixels of the camera. Note that $\overline{C_{\Gamma \rightarrow S}}$ can not be directly obtained from (11) or (12) since it is the ratio of the camera view integrals rather than an integral of the local $C_{\Gamma \rightarrow S}$ values. The same extension for the ionization to photon conversion in (13) gives

$$
\overline{C_{\mathrm{S} / \mathrm{XB}}}=\int_{V_{\text {cam }}} d V \Sigma / \int_{V_{\text {cam }}} d V \varepsilon .
$$

For the global particle balance we require the total recycling flux, which may not be completely contained in the observed area $A_{\text {cam. }}$. Thus, for interpretation of the integrated camera observations, we have to introduce an additional factor for relating the recycling flux on the total limiter surface to the recycling flux within the observed area on the limiter. This factor is mostly a geometric parameter and remains at 2.02 for the given setup with a variation of $1 \%$ over a density increase of one order of magnitude (but may be affected by details of the magnetic configuration and assumptions for cross-field transport). The resulting conversion coefficients $\overline{C_{\Gamma \rightarrow S}}$ and $\overline{C_{\mathrm{S} / \mathrm{XB}}}$ are shown in figure 9. The spatial variations of $C_{\Gamma \rightarrow S}$ and $C_{\mathrm{S} / \mathrm{XB}}$ are averaged out in their integral counterparts but a dependence on plasma conditions remains. At the reference density of $n_{\text {sepx }}=2 \cdot 10^{18} \mathrm{~m}^{-3}$, the integral conversion factor between ionization and recycling in the camera's view cone is still 1.6. As observed in the previous section, this ratio becomes smaller at higher density because ionization becomes more localized in front of the limiter. A larger camera view (green and blue curves in figure 9) may mitigate the density (and implicit temperature) dependence because more of the recycled neutral particles can be captured when they are ionized. However, increasing the camera view increases the emphasis of the periphery of the view where $C_{\mathrm{S} / \mathrm{XB}}$ drops off faster at higher density (as seen in figure $8(\mathrm{a})$ ), and thus introduces a larger variation in $\overline{C_{\mathrm{S} / \mathrm{XB}}}$. The key point is that even the interpretation of the integrated camera observation in terms 
Reconstruction of recycling flux from synthetic camera images, evaluated for the Wendelstein $7-X$ startup lim

of the global particle balance can be challenging without further information about the photon emission and ionization distribution.

\section{Conclusions}

A synthetic diagnostic module has been developed for the EMC3-EIRENE code which can provide valuable information for the interpretation of spectroscopic measurements. Initially developed to generate synthetic camera images (i.e. the forward calculation), it has been extended for evaluation of the inverse problem where information in the (synthetic) camera images is connected back to the originating physical processes. This information is necessary for the reconstruction of the recycling flux from spectroscopic observations. For this inverse problem, we have related the observable photon emission via the ionization of neutral particles within the view cone to the originating recycling flux. The former relation represents a generalization of a local S/XB value to a view cone integral, and the latter relation captures the contributions which go beyond the traditional S/XB framework. Based on modeling results from EMC3-EIRENE, these conversion steps have been analyzed for the W7-X startup limiter.

It has been found that molecular dissociation can contribute significantly to the local $H_{\alpha}$ emission, resulting an factor of 2 increase in the local ionization to

photon conversion $(\mathrm{S} / \mathrm{XB})$. Since modeling provides both the local ionization rates and photon emissions, self consistent $\mathrm{S} / \mathrm{XB}$ values can be calculated from that, i.e. including molecular dissociation effects which are missing in other $\mathrm{S} / \mathrm{XB}$ sources (e.g. from ADAS). Line of sight integration in the conversion of the observed photon emission mitigates this effect to some extent, but a variation across the camera image and a dependence on plasma conditions remains. Furthermore, a spatially resolved reconstruction of recycling is complicated by the non-local nature of the re-ionization of recycled particles, which may occur outside the view cone. It has been found that this effect can lead to an underestimation of the peak recycling flux by a factor of 3-4, while it can also lead to an overestimation in between the peak. These effects are mitigated for an analysis of the global particle balance, because spatial variations in the conversion factors are averaged over the total view cone of the camera. However, a factor of up to 1.6 remains for relevant densities.

Conversion factors have been extracted from simulation results which capture the spatial dependence of the modeled data over a large range of densities. The presented results may guide the interpretation of spectroscopic measurements, which otherwise require assumptions for the recycling to line-of-sight integrated ionization / photon emission conversion. With information on the spatial distribution of ionization and photon emission, dedicated modeling can eliminate these assumptions (although some uncertainties remain due to other assumptions in the edge plasma model) and provide important physics insight. 


\section{Acknowledgements}

This work is supported in part by the U.S. Department of Energy under DE-SC0014210. This work has been carried out within the framework of the EUROfusion Consortium and has received funding from the Euratom research and training programme 20142018 under grant agreement No 633053. The views and opinions expressed herein do not necessarily reflect those of the European Commission.

\section{Appendix A. View cone vs. line of sight integrals}

By resolving the steps from recycling via re-ionization in the camera view cone via photon emission in the view cone to measurable photon emission at the camera location we come across several view cone integrals. We proceed with the reasonable assumption that the resolution of the camera is high enough (otherwise one may split a pixel into small enough virtual sub-pixels and collect their contributions). In this case, the quantity of interest becomes a function of the line of sight coordinate only $(q=q(s))$, and one can write the view cone integral as

$$
\int_{V_{\mathrm{pix}}} d V q=\alpha_{\text {pixel }} \int_{L_{\mathrm{pix}}} d s s^{2} q(s)
$$

with $d V=\delta A \cdot d s$ and $\delta A=\alpha_{\text {pixel }} s^{2}$ (see figure 4). For the measurable photon emission $\Phi_{M}$ we have $q=F(s) \cdot \varepsilon(s)$ where $F(s)=1 / 4 \pi s^{2}$ accounts for the collection solid angle dependency of the incident photons at the position of the camera. The view cone integral then reduces to a standard line of sight integral

$$
\Phi_{M}=\frac{\alpha_{\text {pixel }}}{4 \pi} \int d s \varepsilon(s)=\frac{\alpha_{\text {pixel }}}{4 \pi} \Phi .
$$

That is, the collection angle dependency cancels the $s^{2}$ dependency of the volume element $d V$ (the Etendue of an optical system is constant along the line of sight). On the other hand, the $s^{2}$ dependency is retained in the view cone integrals of the ionization rate and the volumetric photon emission itself. However, one may evaluate the difference between the emitted and measurable photons in terms of a geometry factor

$$
G=\frac{\int_{L} d s s^{2} \varepsilon(s)}{L^{2} \int_{L} d s \varepsilon(s)} .
$$

As long as the emission is located around the target point $s=L$ far away from the camera at $s=0$, one finds $G \approx 1$. For the present diagnostic setup we find $G \approx 1.06$ on the limiter. Nevertheless, a different camera setup may result in larger deviations which would need to be accounted for in the unfolded reconstruction (16). 
Reconstruction of recycling flux from synthetic camera images, evaluated for the Wendelstein $7-X$ startup lim

\section{References}

[1] R. J. Colchin, D. L. Hillis, R. Maingi, C. C. Klepper, and N. H. Brooks. The Filterscope. Rev. Sci. Instrum., 74:2068, 2003.

[2] A. Pospieszczyk. Spectroscopic Diagnostics of Tokamak Edge Plasmas. Phys. Scr., T119:71, 2005.

[3] J. L. Terry and M. L. Reinke. Diagnostic tools for studying divertor detachment: bolometry, spectroscopy, and thermography for surface heat-flux. Plasma Phys. Control. Fusion, 59:044004, 2017.

[4] R. König, N. Ramasubramanian, K. McCormick, P. Grigull, F. Gadelmeier, U. Wenzel, B. Schweer, and M. Brix. Island divertor spectroscopy at the Wendelstein W7-AS stellarator. Rev. Sci. Instrum., 74:2052, 2003.

[5] N. Ramasubramanian, R. König, Y. Feng, L. Giannone, P. Grigull, T. Klinger, K. McCormick, H. Thomsen, U. Wenzel, and the W7-AS Team. Characterization of the island divertor plasma of W7-AS stellarator in the deeply detached state with volume recombination. Nuclear Fusion, 44:922, 2004.

[6] L. Stephey, G. A. Wurden, O. Schmitz, H. Frerichs, F. Effenberg, C. Biedermann, J. Harris, R. König, P. Kornejew, M. Krychowiak, E. A. Unterberg, and W7-X Team. Spectroscopic imaging of limiter heat and particle fluxes and the resulting impurity sources during Wendelstein 7-X startup plasmas. Rev. Sci. Intrum., 87:11D606, 2016.

[7] G. A. Wurden, L. A. Stephey, C. Biedermann, M. W. Jakubowski, J. P. Dunn, M. Gamradt, and W7-X Team. A high resolution IR/visible imaging system for the W7-X limiter. Rev. Sci. Intrum., 87:11D607, 2016.

[8] M. Goto, K. Sawada, T. Oishi, and S. Morita. Particle source and edge confinement study based on spectroscopic diagnosis in the LHD. Plasma Phys. Control. Fusion, 58:084001, 2016.

[9] M. Kobayashi, S. Morita, and M. Goto. Space-resolved visible spectroscopy for two-dimensional measurement of hydrogen and impurity emission spectra and of plasma flow in the edge stochastic layer of LHD. Rev. Sci. Instrum., 88:033501, 2017.

[10] S. P. Gerhardt, J. M. Canik, D. T. Anderson, and L. Owen. $\mathrm{H}_{\alpha}$ detector system for the Helically Symmetric Experiment. Rev. Sci. Instrum., 75:2981, 2004.

[11] J. M. Canik, D. T. Anderson, F. S. B. Anderson, C. Clark, K. M. Likin, J. N. Talmadge, and K. Zhai. Reduced particle and heat transport with quasisymmetry in the Helically Symmetric Experiment. Phys. Plasmas, 14:056107, 2007.

[12] R. C. Isler, R. W. Wood, C. C. Klepper, N. H. Brooks, M. E. Fenstermacher, and A. W. Leonard. Spectroscopic characterization of the DIII-D divertor. Phys. Plasmas, 4:355, 1997.

[13] E. M. Hollmann, S. Brezinsek, N. H. Brooks, M. Groth, A. G. McLean, A. Yu. Pigarov, and D. L. Rudakov. Spectroscopic measurement of atomic and molecular deuterium fluxes in the DIII-D plasma edge. Plasma Phys. Control. Fusion, 48:1165, 2006.

[14] V. A. Soukhanovskii, A. L. Roquemore, C. H. Skinner, J. Menard, H. W. Kugel, D. Johnson, R. Maingi, S. Sabbagh, and F. Paoletti. High-resolution spectroscopic diagnostic for divertor and scrape-off layer neutral and impurity emission measurements in the National Spherical Torus Experiment. Rev. Sci. Instrum., 74:2094, 2003.

[15] J-W. Ahn, K.F. Gan, F. Scotti, J.D. Lore, R. Maingi, J.M. Canik, T.K. Gray, A.G. McLean, A.L. Roquemore, and V.A. Soukhanovskii. Study of non-axisymmetric divertor footprints using 2-D IR and visible cameras and a 3-D heat conduction solver in NSTX. J. Nucl. Mater., 438:S317, 2013.

[16] S. Brezinsek, G. Sergienko, A. Pospieszczyk, Ph. Mertens, U. Samm, and P. T. Greenland. Characterization of the deuterium recycling flux in front of a graphite surface in the TEXTOR tokamak. Plasma Phys. Control. Fusion, 47:615, 2005.

[17] M. Clever, S. Brezinsek, H. Frerichs, M. Lehnen, A. Pospieszczyk, D. Reiter, U. Samm, O. Schmitz, B. Schweer, and the TEXTOR-Team. Experimental investigation of density regimes in the helical divertor at TEXTOR. Nuclear Fusion, 52:054005, 2012. 
[18] K. Behringer, H. P. Summers, B. Denne, M. Forrest, and M. Stamp. Spectroscopic determination of impurity influx from localized surfaces. Plasma Phys. Control. Fusion, 31:2059, 1989.

[19] Y. Feng, F. Sardei, and J. Kisslinger. 3D fluid modelling of the edge plasma by means of a Monte Carlo technique. Journal of Nuclear Materials, 266-269:812-818, 1999.

[20] Y. Feng, H. Frerichs, M. Kobayashi, A. Bader, F. Effenberg, D. Harting, H. Hoelbe, J. Huang, G. Kawamura, J. D. Lore, T. Lunt, D. Reiter, O. Schmitz, and D. Sharma. Recent Improvements in the EMC3-Eirene Code. Contrib. Plasma Phys., 54(4-6):426-431, 2014.

[21] Y. Feng, F. Sardei, P. Grigull, K. McCormick, J. Kisslinger, D. Reiter, and Y. Igitkhanov. Transport in island divertors: physics, 3D modelling and comparison to first experiments on W7-AS*. Plasma Phys. Control. Fusion, 44:611-625, 2002.

[22] Y. Feng, F. Sardei, J. Kisslinger, P. Grigull, K. McCormick, D. Reiter, L. Giannone, R. König, N. Ramasubramanian, H. Thomsen, and U. Wenzel. Physics of the geometry-related detachment stability in W7-AS. Nuclear Fusion, 45:89-95, 2005.

[23] Y. Feng, F. Sardei, P. Grigull, K. McCormick, J. Kisslinger, and D. Reiter. Physics of island divertors as highlighted by the example of W7-AS. Nuclear Fusion, 46:807-819, 2006.

[24] Y. Feng, F. Sardei, and J. Kisslinger. A simple highly accurate field-line mapping technique for three-dimensional Monte Carlo modeling of plasma edge transport. Phys. Plasmas, 12(052505):1-7, 2005.

[25] H. Frerichs, D. Reiter, Y. Feng, and D. Harting. Block-structured grids in Lagrangian 3D edge plasma transport simulations. Comp. Phys. Commun., 181:61-70, 2010.

[26] M. Kobayashi, Y. Xu, K. Ida, Y. Corre, Y. Feng, O. Schmitz, H. Frerichs, F.L. Tabares, T.E. Evans, J.W. Coenen, Y. Liang, A. Bader, K. Itoh, H. Yamada, Ph. Ghendrih, G. Ciraolo, D. Tafalla, A. Lopez-Fraguas, H.Y. Guo, Z.Y. Cui, D. Reiter, N. Asakura, U. Wenzel, S. Morita, N. Ohno, B.J. Peterson, and S. Masuzaki. 3D effects of edge magnetic field configuration on divertor/scrape-off layer transport and optimization possibilities for a future reactor. Nuclear Fusion, 55(10):104021, 2015.

[27] Y. Feng, C.D. Beidler, J. Geiger, P. Helander, H. Hölbe, H. Maassberg, Y. Turkin, D. Reiter, and W7-X Team. On the W7-X divertor performance under detached conditions. Nuclear Fusion, $56: 126011,2016$.

[28] F. Effenberg, Y. Feng, O. Schmitz, H. Frerichs, S. Bozhenkov, H. Hölbe, R. König, M. Krychowiak, T. Sunn Pedersen, D. Reiter, L. Stephey, and the W7-X Team. Numerical investigation of plasma edge transport and limiter heat fluxes in Wendelstein 7-X startup plasmas with EMC3-Eirene. Nuclear Fusion, 57:036021, 2017.

[29] G.A. Wurden, C. Biedermann, F. Effenberg, M. Jakubowski, H. Niemann, L. Stephey, S. Bozhenkov, S. Brezinsek, J. Fellinger, B. Cannas, F. Pisano, S. Marsen, H.P. Laqua, R. König, O. Schmitz, J.H. Harris, E.A. Unterberg, and the W7-X Team. Limiter observations during W7$\mathrm{X}$ first plasmas. Nuclear Fusion, 57:056036, 2017.

[30] T. Sunn Pedersen, M. Otte, S. Lazerson, P. Helander, S. Bozhenkov, C. Biedermann, T. Klinger, R.C. Wolf, H.-S. Bosch, and The Wendelstein 7-X Team. Confirmation of the topology of the Wendelstein 7-X magnetic field to better than 1:100,000. Nature Communications, 7, 2016.

[31] S.A. Lazerson, M. Otte, S. Bozhenkov, C. Biedermann, T. Sunn Pedersen, and the W7-X Team. First measurements of error fields on W7-X using flux surface mapping. Nuclear Fusion, 56:106005, 2016.

[32] T. Sunn Pedersen, T. Andreeva, H.-S. Bosch, S. Bozhenkov, F. Effenberg, M. Endler, Y. Feng, D.A. Gates, J. Geiger, D. Hartmann, H. Hölbe, M. Jakubowski, R. König, H.P. Laqua, S. Lazerson, M. Otte, M. Preynas, O. Schmitz, T. Stange, Y. Turkin, and the W7-X Team. Plans for first plasma operation of Wendelstein 7-X. Nuclear Fusion, 55:126001, 2015.

[33] Y. Feng, F. Sardei, J. Kisslinger, P. Grigull, K. McCormick, and D. Reiter. 3D Edge Modeling and Island Divertor Physics. Contrib. Plasma Phys., 44(1-3):57-69, 2004.

[34] H. Frerichs. 3D plasma transport in open chaotic magnetic fields: A computational assessment for tokamak edge layers. Technical Report Jül-4321, Berichte des Forschungszentrum Jülich, 2010. 
Reconstruction of recycling flux from synthetic camera images, evaluated for the Wendelstein $7-X$ startup lim

ISSN 0944-2952.

[35] D. Reiter, M. Baelmans, and P. Boerner. The EIRENE and B2-EIRENE codes. Fusion Science and Technology, 47(2):172, 2005.

[36] D. Reiter. EIRENE - A Monte Carlo linear transport solver. http://www.eirene.de.

[37] W. Eckstein. Data sets for hydrogen reflection and their use in neutral transport calculations. J. Nucl. Mater., 145-147:332, 1987.

[38] The AMJUEL online database. http://www.eirene.de/html/amjuel.html.

[39] K. Sawada and T. Fujimoto. Effective ionization and dissociation rate coefficients of molecular hydrogen in plasma. J. Appl. Phys., 78((5)):2913, 1995.

[40] M. Krychowiak, A. Adnan, A. Alonso, T. Andreeva, J. Baldzuhn, T. Barbui, M. Beurskens, W. Biel, C. Biedermann, B. D. Blackwell, H. S. Bosch, S. Bozhenkov, R. Brakel, T. Bräuer, B. Brotas de Carvalho, R. Burhenn, B. Buttenschön, A. Cappa, G. Cseh, A. Czarnecka, A. Dinklage, P. Drews, A. Dzikowicka, F. Effenberg, M. Endler, V. Erckmann, T. Estrada, O. Ford, T. Fornal, H. Frerichs, G. Fuchert, J. Geiger, O. Grulke, J. H. Harris, H. J. Hartfus̈, D. Hartmann, D. Hathiramani, M. Hirsch, U. Höfel, S. Jablonski, M. W. Jakubowski, J. Kaczmarczyk, T. Klinger, S. Klose, J. Knauer, G. Kocsis, R. König, P. Kornejew, A. KrämerFlecken, N. Krawczyk, T. Kremeyer, I. Ksiazek, M. Kubkowska, A. Langenberg, H. P. Laqua, M. Laux, S. Lazerson, Y. Liang, S. C. Liu, A. Lorenz, A. O. Marchuk, S. Marsen, V. Moncada, D. Naujoks, H. Neilson, O. Neubauer, U. Neuner, H. Niemann, J. W. Oosterbeek, M. Otte, N. Pablant, E. Pasch, T. Sunn Pedersen, F. Pisano, K. Rahbarnia, L. Ryc, O. Schmitz, S. Schmuck, W. Schneider, T. Schröder, H. Schuhmacher, B. Schweer, B. Standley, T. Stange, L. Stephey, J. Svensson, T. Szabolics, T. Szepesi, H. Thomsen, J.-M. Travere, H. Trimino Mora, H. Tsuchiya, G. M. Weir, U. Wenzel, A. Werner, B. Wiegel, T. Windisch, R. Wolf, G. A. Wurden, D. Zhang, A. Zimbal, S. Zoletnik, and W7-X Team. Overview of diagnostic performance and results for the first operation phase in Wendelstein 7-X (invited). Rev. Sci. Intrum., 87:11D304, 2016 .

[41] H. Frerichs, F. Effenberg, O. Schmitz, C. Biedermann, Y. Feng, M. Jakubowski, R. König, M. Krychowiak, J. Lore, H. Niemann, T. Sunn Pedersen, L. Stephey, G. A. Wurden, and the W7-X Team. Synthetic plasma edge diagnostics in EMC3-EIRENE, highlighted for Wendelstein 7-X (W7-X). Rev. Sci. Instrum., 87:11D441, 2016.

[42] H. Frerichs, D. Reiter, M. Clever, and Y. Feng. Three-dimensional computer simulations of local and line-of-sight integrated $H_{\alpha}$ line emission in the TEXTOR helical divertor. In 37th EPS Conference on Plasma Physics, number P2.127, 2010.

[43] H. P. Summers, W. J. Dickson, M. G. O’Mullane, N. R. Badnell, A. D. Whiteford, D. H. Brooks, J. Lang, S. D. Loch, and D. C. Griffin. Ionization state, excited populations and emission of impurities in dynamic finite density plasmas: I. The generalized collisional-radiative model for light elements. Plasma Phys. Control. Fusion, 48:263-293, 2006.

[44] OPEN-ADAS - Atomic Data and Analysis Structure: open.adas.ac.uk. 